\title{
EXPERIENCIAS DE APROVECHAMIENTO DE RESIDUOS PARA LA GENERACIÓN DE BIODIÉSEL EN COLOMBIA Y MÉXICO
}

\author{
Gisela MONTERO ALPÍREZ ${ }^{1 *}$, Beatriz E. JARAMILLO COLORADO², Ana M. VÁZQUEZ ESPINOZA3, \\ Marcos A. CORONADO ORTEGA ${ }^{1}$, Conrado GARCÍA GONZÁLEZ ${ }^{1}$ y Lydia TOSCANO PALOMAR ${ }^{4}$
}

${ }^{1}$ Instituto de Ingeniería, Universidad Autónoma de Baja California, Calle de la Normal S/N, Col. Insurgentes Este, CP 21280, Mexicali, BC, México

${ }^{2}$ Facultad de Ciencias Exactas y Naturales, Universidad de Cartagena, Av. del Consulado, Calle 30 No. 48 152, Cartagena, Bolívar, Colombia

${ }^{3}$ Escuela de Ingeniería y Negocios, Guadalupe Victoria, Universidad Autónoma de Baja California Carretera Estatal no.3 Col. Gutiérrez s/n. CP 21720, delegación Guadalupe Victoria, Mexicali, BC., México

${ }^{4}$ Academia de Química y Bioquímica, Instituto Tecnológico de Mexicali, Av. Tecnológico S/N, Col. Elías Calles, Mexicali, B.C., CP 21396, México

*Autor para correspondencia: gmontero@uabc.edu.mx

(Recibido septiembre 2014; aceptado junio 2015)

Palabras clave: residuos oleicos, biodiésel, transesterificación

\section{RESUMEN}

En la búsqueda de alternativas al uso de combustibles fósiles, se han desarrollado diversas tecnologías para obtener biocombustibles. Entre ellos se encuentra el biodiesel, que puede ser obtenido de aceites comestibles de cultivos oleaginosos; de cultivos que no compiten con alimentos o mejor aún, de residuos oleosos generados a partir de actividades agrícolas, pecuarias y urbanas. Con la aprobación en 2001, de la Ley 693 "Usos de Alcoholes Carburantes", Colombia inició la era de los biocombustibles y actualmente cuenta con nueve plantas de producción de biodiesel a partir de aceite de palma. En México, en 2008 entró en vigor la Ley de Promoción y Desarrollo de los Bioenergéticos. Ello sentó las bases para la producción de biodiesel a nivel industrial en el país. En México se instalaron seis plantas distribuidas en los estados de Chiapas, Michoacán y Nuevo León, capaces de procesar aceite de palma, Jatropha Curcas L., higuerilla, aceites vegetales residuales y sebo animal. Es así que este trabajo muestra un análisis comparativo del desarrollo de la industria del biodiesel en Colombia y México, considerando la utilización de residuos. De acuerdo con los resultados, para producir biodiesel en Colombia se utiliza una tecnología que procesa palma la cual genera residuos que son aprovechados como fuente de energía en otros procesos. Mientras que el uso de residuos para obtener biodiesel se encuentra en etapa de investigación. En México, las tecnologías de las plantas fueron diseñadas para procesar residuos y semillas oleaginosas. Estas últimas generan residuos que no son reutilizados.

Key words: oleic wastes, biodiesel, transesterification

\begin{abstract}
In the search for alternatives to fossil fuels, diverse technologies for the production of biofuels have been developed. Among them is biodiesel, which can be obtained from oil seeds or oil residues generated from agriculture, livestock and urban activities. With the
\end{abstract}


approval in 2001 of Act-693 "Uses of Alcohol Fuels", Colombia began the era of biofuels and currently has nine biodiesel production plants from palm oil. In the case of Mexico, in 2008 the Law on Promotion and Development of Bioenergetics was enacted. This laid the foundation for the production of biodiesel on an industrial level nationally. In Mexico, six biodiesel plants were installed in the states of Chiapas, Michoacán and Nuevo León capable of processing, Jatropha Curcas L., palm oil, castor oil, waste vegetable oil and animal tallow. Therefore, the main objective of this paper is to show a comparative analysis of the development of the biodiesel industry in Colombia and Mexico, considering the use of waste. According to the results, the biodiesel production in Colombia is based on a technology that processes palm which generates waste. The latter is used as an energy source in other processes. The use of waste for biodiesel is under investigation. In Mexico, the technologies of the plants were designed to process waste and oil seeds. These oil seeds generate residues that are not reused.

\section{INTRODUCCIÓN}

El compromiso con el desarrollo sustentable incluye el aprovechamiento de las energías renovables, debido a que presentan un bajo impacto ambiental, contribuyen a mitigar el cambio climático y reducen la dependencia hacia los combustibles fósiles. Asimismo han servido para promover la producción y uso de los biocombustibles a nivel mundial. Los biocombustibles se derivan de la biomasa y pueden estar en estado sólido, líquido o gaseoso. La biomasa residual también es utilizada para la producción de biocombustibles.

De acuerdo con IEA (2014), los factores clave para la introducción y establecimiento de políticas internacionales que brinden soporte a los biocombustibles son: a) desarrollo del campo y comunidades rurales, b) seguridad energética, c) reducción de la dependencia al petróleo y d) mitigación del cambio climático. Hay países que ya han establecido la obligatoriedad del consumo de biocombustibles a través de sus políticas y leyes. Otros países únicamente lo han considerado como parte de sus objetivos para la transición energética, sin determinar la obligatoriedad. La figura 1 muestra un panorama global de los países que ya cuentan con mandatos en cuanto al uso de biocombustibles, y aquéllos que solamente tienen como objetivo el desarrollo de biocombustibles.

Brasil y Estados Unidos fueron los primeros países en establecer programas de biocombustibles en 1970 's/80's. Estados Unidos introdujo en 2003, un mandato para utilizar $5.75 \%$ de biocombustibles en el sector transporte en 2010. Posteriormente, se modificó este mandato para incrementar la participación hasta $10 \%$ en 2020, como energía renovable. A la fecha, más de 50 países cuentan con mandatos para la producción y uso de biocombustibles, tales como el bioetanol y el biodiesel.
En la actualidad, la producción de biodiesel en el contexto internacional presenta una tendencia hacia el alza, esto como resultado de los esfuerzos globales para mitigar la problemática de cambio climático y promover el desarrollo sustentable, como se puede observar en la figura 2 (adaptado de IRENA 2013).

La unión europea se posiciona como la región con mayor participación y dinámica en la producción de biodiesel globalmente, seguida de Estados Unidos, Argentina, Brasil, Indonesia, Singapur, Tailandia y otros países.

En la categoría de otros países se encuentra Colombia que se posiciona con uno de los mercados más dinámicos en la producción y comercialización de biodiesel de palma. Mientras que México, cuenta con un alto potencial de producción de biodiesel a partir de cultivos no alimenticios y de residuos vegetales y animales.

Existen varias razones por las cuales es apropiado promover el desarrollo del biodiesel a partir de residuos biomásicos, entre las cuales se mencionan las siguientes (Canacki y Gerpen 1999, Gerpen et al. 2006):

- Representa un método sustentable en el cual se revaloriza y reutiliza un recurso con un alto contenido energético para producir un combustible más limpio. De esta manera no se desaprovecha un recurso valioso desde el punto de vista energético, y al mismo tiempo el ambiente y la sociedad en general son beneficiados.

- Evita el uso de aceites comestibles en la producción de biodiesel, por ello no pone en riesgo la seguridad y soberanía alimentaria porque se reutilizan residuos.

- Representa una oportunidad para mitigar el impacto ambiental causado por las emisiones de gases de efecto invernadero, responsables del calentamiento global. 


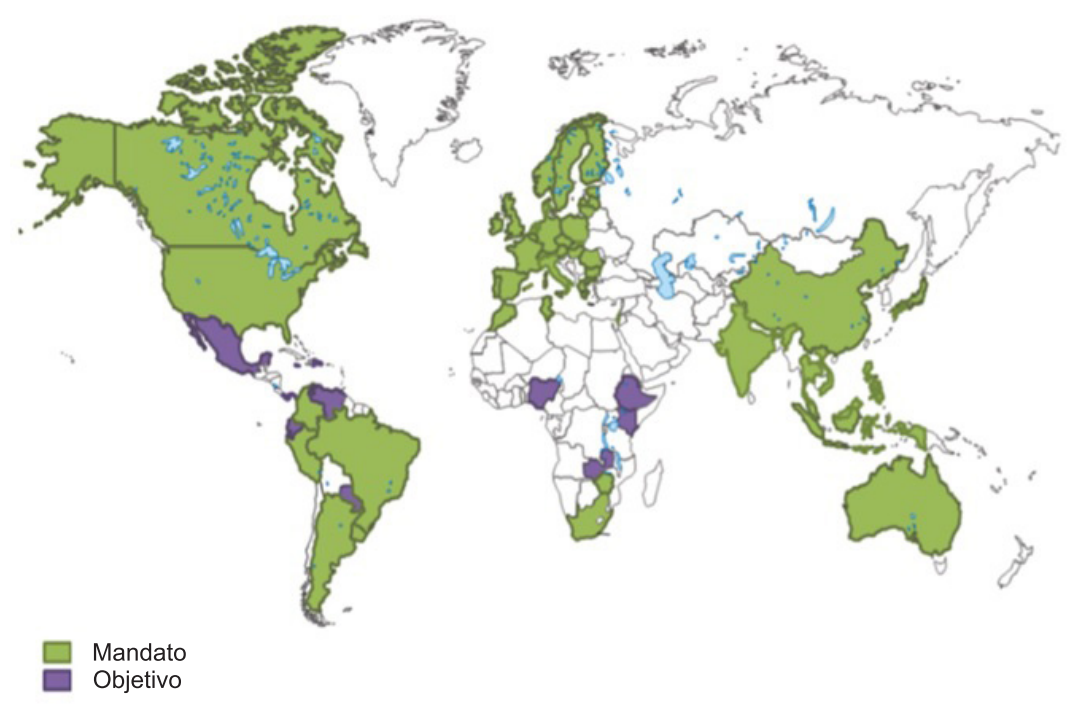

Fig. 1. Países con políticas internacionales de soporte a los biocombustibles (IEA 2014).

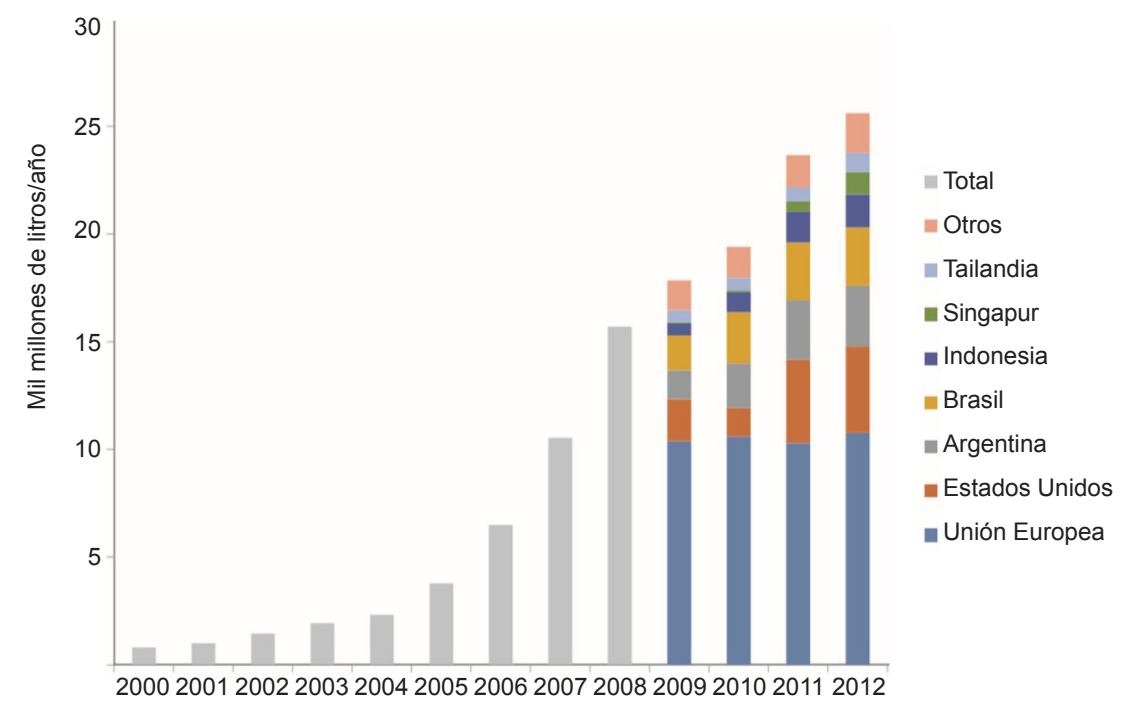

Fig. 2. Produción global de biodiesel

- Es una oportunidad para diversificar la matriz energética, tradicionalmente basada en combustibles fósiles.

- Reduce la dependencia de los combustibles fósiles.

- Las emisiones de dióxido de carbono están integradas al ciclo de carbono de las plantas, de las cuales se extraen los aceites.

- Tiene excelentes propiedades lubricantes para su uso en los motores diesel.

- Proviene de una fuente renovable.

- Es biodegradable.
La importancia del biodiesel se ha señalado en obras recientes, como un combustible alternativo para el motor diesel (Graboski y McCormick 1998, Canacki 2007, Lin et al. 2006, Rakopoulos et al. 2006, Altin et al. 2001, Iscan y Aydin 2012).

La American Society of Testing and Materials (ASTM), define al biodiesel como una mezcla de ésteres mono alquílicos de ácidos grasos de cadena larga, obtenidos de aceites vegetales o grasas animales y se utilizan en los motores de ignición compresión (diesel) (ASTM D6751). 


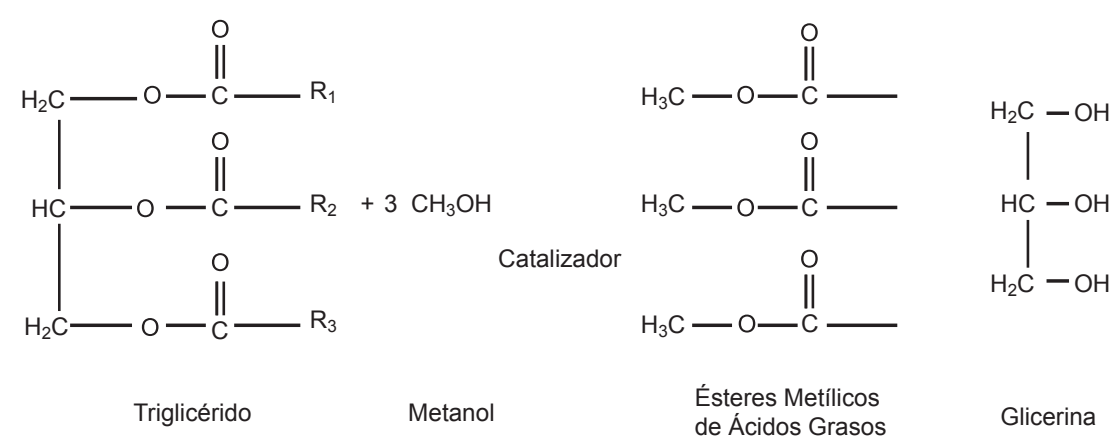

Fig. 3. Reacción de transesterificación para obtener biodiesel

El biodiesel se obtiene mediante la transesterificación de aceites de origen animal o vegetal, es decir, triglicéridos con un alcohol de cadena corta en presencia de un catalizador, de acuerdo con la siguiente reacción ilustrada en la figura 3.

Esta reacción puede ser catalizada por álcalis, ácidos (Canacki y Gerpen 1999, Vyas et al. 2010) o enzimas (Nielsen et al. 2008). Se han realizado varios estudios utilizando diferentes aceites como materia prima, distintos alcoholes, al igual que una variedad de catalizadores, incluyendo catalizadores homogéneos como el hidróxido de sodio, hidróxido de potasio y ácido sulfúrico, y catalizadores heterogéneos como las lipasas (Nielsen et al. 2008, Boey et al. 2009, Refaat 2011).

El biodiesel es una alternativa al diesel y se obtiene a partir de la biomasa, por ello es de carácter renovable. Dependiendo de la fuente de materia prima se clasifica como: primera, segunda o tercera generación. Se entiende como biodiesel de primera generación aquél que se produce del procesamiento de aceites extraídos de cultivos alimenticios, e.g. aceites de soya, girasol y canola.

Se denomina segunda generación cuando proviene de cultivos que no compiten con alimentos o de residuos animales y vegetales, e.g. Jatropha curcas $L$., higuerilla, grasas amarillas, sebos animales y aceites vegetales residuales (AVR). Y por último, de tercera generación al ser producido a partir de microalgas.

El presente trabajo muestra un análisis comparativo del desarrollo de la industria del biodiesel en México y Colombia, considerando la utilización de residuos. Colombia cuenta con nueve plantas productoras de biodiesel a escala industrial, a partir de aceite de palma, que se encuentran en operación (FEDEBIOCOMBUSTIBLES 2014). En México existen seis plantas de capacidad industrial localizadas en los estados de Chiapas, Michoacán y Nuevo León.

\section{POLÍTICAS Y MARCO REGULATORIO EN COLOMBIA Y MÉXICO}

Como puede apreciarse en el cuadro I, el nivel de desarrollo que presenta Colombia en cuanto a producción de biodiesel aventaja al de México (Trejo 2007, DOF 2008, DOF 2009). Esto se debe a que en 2001, Colombia entró en la nueva era mundial de los biocombustibles mediante la aprobación de la Ley 693 "Usos de Alcoholes Carburantes", con el atractivo económico de la dinámica de precios internacionales del petróleo y los lineamientos del Protocolo de Kyoto (Ministerio de Agricultura y Desarrollo Rural, República de Colombia 2012, FAO 2013).

El marco regulatorio vigente en Colombia incluye mandatos obligatorios desde 2005, que corresponden a un volumen de biocombustibles del $10 \%$ en las mezclas, tanto para el bioetanol como el biodiesel. Se esperaba que el porcentaje de biodiesel llegue a $20 \%$ en 2012 (FEDEBIOCOMBUSTIBLES 2014). Los programas de promoción también incluyen incentivos a la producción y comercialización, tales como exenciones tributarias tanto a la producción de materias primas como al uso final, la creación de zonas francas para la producción de materias primas y créditos blandos a la inversión. En 2008, se crearon lineamientos con la finalidad de promover estrategias para la producción sustentable de biocombustibles (Duffey 2011).

El marco normativo mexicano es sumamente reciente, se inició en 2008 con la promulgación de la Ley de Promoción y Desarrollo de Bioenergéticos (LPDB), como parte de los compromisos establecidos 
CUADRO I. DERECHO COMPARADO SOBRE BIOCOMBUSTIBLES EN COLOMBIA Y MÉXICO

\begin{tabular}{|c|c|c|}
\hline Año & País & Acción \\
\hline 2001 & Colombia & $\begin{array}{l}\text { Ley } 693 \text { Usos de Alcoholes Carburantes. Iniciativa del proyecto } \\
\text { biogasolina, cuya meta era utilizar una mezcla de } 10 \% \text { de bioetanol } \\
\text { con gasolina. }\end{array}$ \\
\hline 2004 & Colombia & $\begin{array}{l}\text { Ley } 939 \text { Biodiesel. En su artículo } 8^{\circ} \text { establece que el biocombustible } \\
\text { de origen vegetal o animal para uso en motores diesel de produc- } \\
\text { ción nacional con destino a la mezcla con diesel estará exento del } \\
\text { impuesto a las ventas y en su artículo } 9^{\circ} \text { establece su exención al } \\
\text { impuesto global del diesel. }\end{array}$ \\
\hline 2005 & Colombia & $\begin{array}{l}\text { Mandato obligatorio para utilizar mezclas de } 10 \% \text { de bioetanol en } \\
\text { las gasolinas y } 10 \% \text { de biodiesel en el diesel. }\end{array}$ \\
\hline 2007 & Colombia & $\begin{array}{l}\text { Decreto } 2629 \text { amplia la mezcla obligatoria de biocombustibles al } \\
10 \% \text { a partir del año } 2010 \text { y establece que los vehículos nuevos } \\
\text { deben ser capaces de consumir una mezcla de al menos } 20 \% \text {. }\end{array}$ \\
\hline 2008 & México & $\begin{array}{l}\text { La Ley DOF-01-02-2008, Ley de Promoción y Desarrollo de los } \\
\text { Bioenergéticos. }\end{array}$ \\
\hline 2009 & Colombia & $\begin{array}{l}\text { El decreto } 1135 / 2009 \text { señala que a partir del } 1 \text { de enero de } 2012 \text {, el } \\
60 \% \text { de los autos vendidos en el país deben ser tipo Flex-Fuel } 85 \text {; } \\
\text { debe alcanzar el } 80 \% \text { en } 2013 \text { y } 100 \text { en } 2014 \text {. }\end{array}$ \\
\hline 2009 & México & $\begin{array}{l}\text { El Reglamento de la Ley de Promoción y Desarrollo de los Bioener- } \\
\text { géticos, emitido el } 18 \text { de junio de 2009, establece las directrices so- } \\
\text { bre permisos relacionados con la aplicación y uso de la referida Ley. }\end{array}$ \\
\hline
\end{tabular}

en los Tratados internacionales que ha firmado, tales como la Convención Marco de Naciones Unidas sobre el Cambio Climático y el Protocolo de Kyoto (DOF 2008). La LPDB tiene como objeto promocionar y desarrollar los bioenergéticos, con la intención de diversificar la matriz energética con base en un desarrollo sustentable sin poner en riesgo la seguridad y soberanía alimentaria del país.

Asimismo, existe el Reglamento de la Ley de Promoción y Desarrollo de los Bioenergéticos (2009) donde se establece que la Secretaría de Energía (SENER) es la encargada de emitir los permisos de producción, almacenamiento, distribución por ductos así como la comercialización de bioenergéticos. Estos permisos son otorgados para realizar las actividades y procesos necesarios, con la finalidad de producir biocombustibles mediante la transformación de la biomasa, proveniente de materia orgánica de las actividades agrícola, pecuaria, silvícola, forestal, acuacultura, algacultura, residuos de la pesca, residuos domésticos, residuos comerciales, residuos industriales, de microorganismos y de enzimas, así como de sus derivados.

En 2014, se presentó un cambio radical en el sector energético a través de la Reforma Energética que permitió la apertura del mercado energético mexicano. Sin embargo, solo dio viabilidad al mercado de los hidrocarburos de origen fósil y a la generación y cogeneración de electricidad a partir de fuentes renovables, principalmente eólica y solar, con un impulso marginal a la utilización de biomasa como insumo para los biocombustibles, sólidos y gaseosos dejando muy rezagados a los biocombustibles líquidos.

Es por ello, que el 15 de diciembre de 2014, se propuso la iniciativa que reforma y adiciona diversas disposiciones de la LPDB a fin de precisar y garantizar que los biocombustibles renovables y los insumos tengan un proceso claro de incorporación y que coadyuven a disminuir de manera clara y con metas obligatorias la dependencia de fuentes de energía fósiles y que ésta sea de bajas emisiones de $\mathrm{CO}_{2} \mathrm{y}$ que promueva el desarrollo del sector rural (Gaceta Parlamentaria 2014).

La iniciativa argumentó que la LPDB:

- Se orienta como una ley que promueve el desarrollo de los bioenergéticos

- No establece objetivos y metas obligatorias para su cumplimiento

- Carece de aplicación práctica

- No establece tiempos y porcentajes de cumplimiento obligatorio de cada uno de los actores que conforman la cadena de valor de los bioenergéticos y biocombustibles 


\section{PLANTAS PRODUCTORAS DE BIODIESEL EN COLOMBIA}

Colombia cuenta con nueve plantas productoras de biodiesel de primera generación, a partir de aceite de palma que se encuentran en operación localizadas en los Departamentos de: Atlántico, Cesar, Cundinamarca, Magdalena, Meta y Santander como se ilustra en la figura 4. Las nueve plantas suman en total una capacidad instalada de 591,000 t/año (FEDEBIOCOMBUSTIBLES 2014).
El cuadro II resume la información de las plantas instaladas en Colombia. Se puede apreciar que a partir desde 2008 entraron en operación plantas productoras de biodiesel.

La palma aceitera constituye el cultivo principal en Colombia para la producción de biodiesel. En 2012, se reportaron 299,953 ha de superficie sembrada de palma en producción y 152,482 ha sembradas de palma en desarrollo (FEDEBIOCOMBUSTIBLES 2014). Con base en el tipo de materia prima utilizada, se trata de biodiesel denominado de primera generación.

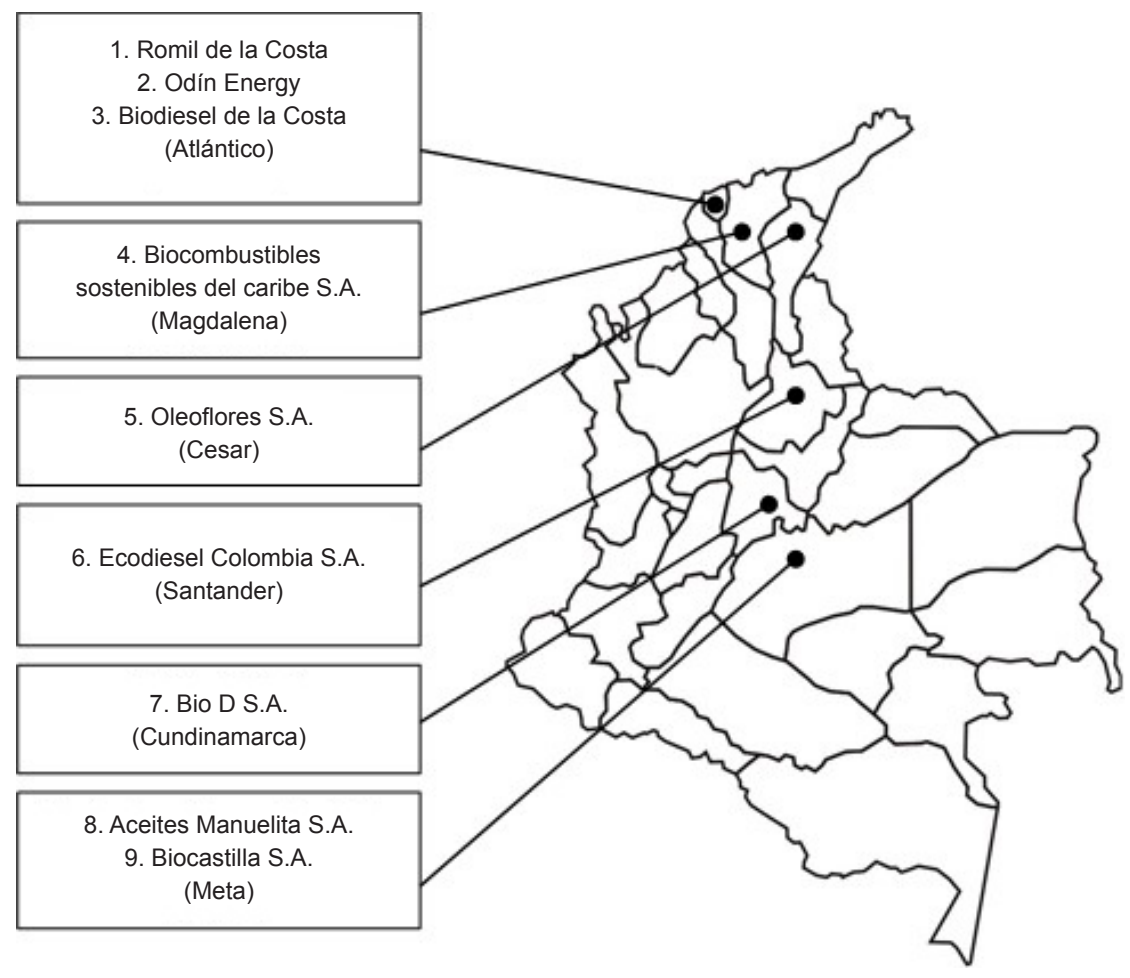

Fig. 4. Ubicación de plantas productoras de biodiesel en Colombia (Elaboración propia)

CUADRO II. INFORMACIÓN DE PLANTAS INSTALADAS EN COLOMBIA

\begin{tabular}{|c|c|c|c|}
\hline Ubicación & Empresa & Capacidad (t/año) & Inicio de operaciones \\
\hline Norte, Santa Marta & $\begin{array}{c}\text { Biocombustibles Sostenibles del } \\
\text { Caribe }\end{array}$ & 100,000 & 2009 \\
\hline Norte, Codazzi & Oleoflores & 60,000 & 2008 \\
\hline Norte, Barranquilla & Romil de la Costa & 10,000 & - \\
\hline Norte, Gálapa & Biodiesel de la Costa & 10,000 & - \\
\hline Norte, Santa Marta & Odín Energy & 36,000 & - \\
\hline Oriental, Facatativá & BioD & 120,000 & 2009 \\
\hline Central, Barrancabermeja & Ecodiesel de Colombia & 120,000 & 2008 \\
\hline Oriental, San Carlos de Guaroa & Aceites Manuelita & 120,000 & 2009 \\
\hline Oriental, Castilla la Grande & Biocastilla & 15,000 & - \\
\hline
\end{tabular}




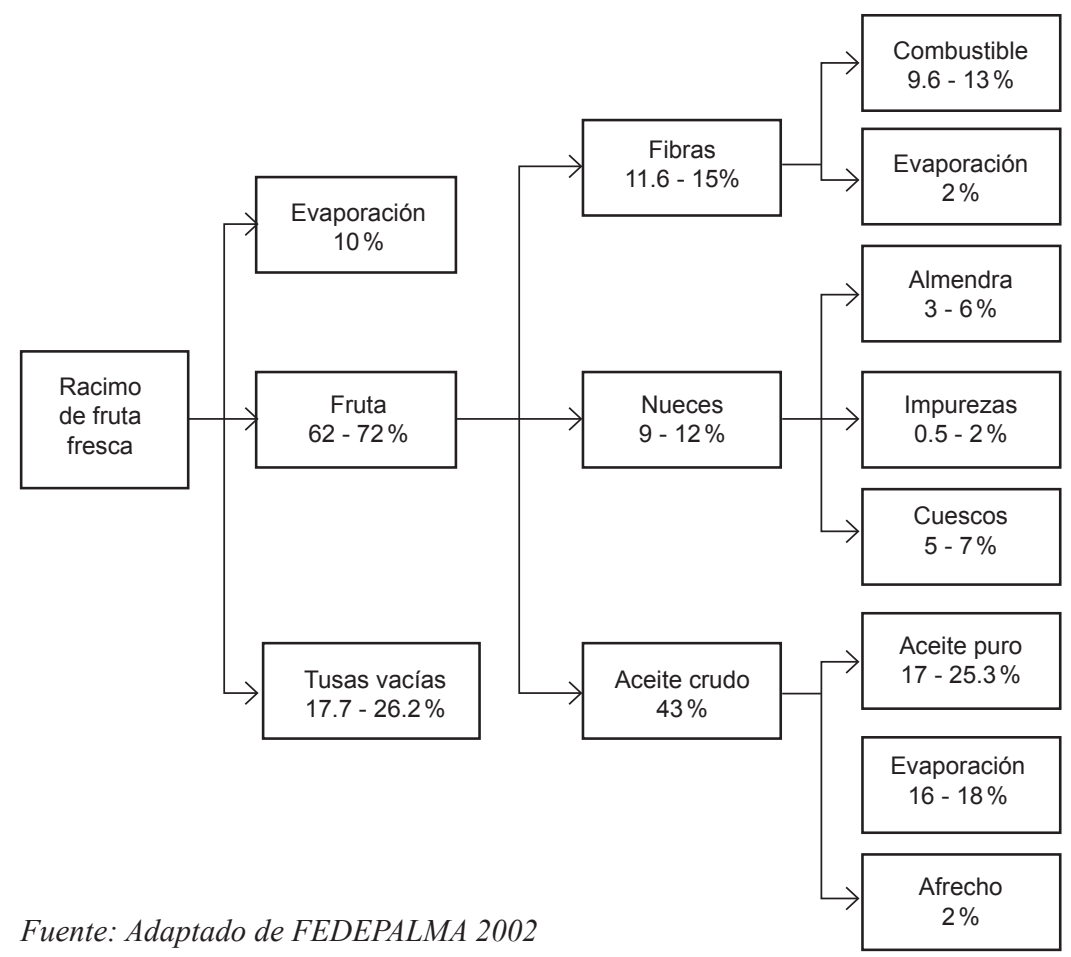

Fig. 5. Balance de masa proceso de extracción de aceite de palma

En el procesamiento de obtención de aceite de palma se generan residuos como se ilustra en la figura 5 (Adaptado de FEDEPALMA 2002). La fibra es utilizada como combustible en calderas para la generación de vapor. La almendra es triturada para la extracción de aceite y la torta resultante es procesada para elaborar harinas proteicas que sirven como alimento para ganado (Reinosa 2009).

\section{PLANTAS PRODUCTORAS DE BIODIESEL EN MÉXICO}

México cuenta con seis plantas productoras de biodiesel de capacidad industrial localizadas en los estados de Chiapas, Michoacán y Nuevo León, tal como se ilustra en la figura 6, las cuales fueron diseñadas para procesar aceite de palma, Jatropha curcas L., higuerilla, AVR y sebo animal, es decir, biodiesel de primera y de segunda generación.

La planta ubicada en Cadereyta, Nuevo León, inició operaciones en 2004. Sin embargo, operó a capacidad completa hasta el año 2009 y parte de 2010, en los cuales tuvo un contrato de venta de biodiesel con PEMEX, quien lo utilizaba como aditivo para mejorar la lubricidad del diesel (Prehn y Cumana 2010). Ante la falta de mercado, esta planta dejó de producir biodiesel, por lo que se adecuó y se dividió en dos módulos: uno para producir combustible para flama abierta y otro para asfalto (El Economista 2011).

En 2006, la empresa MORECO, ubicada en Michoacán, inició un proyecto para recolectar aceites vegetales y grasas animales de desecho para su posterior conversión en biodiesel. Actualmente MORECO recolecta dichos residuos generados en restaurantes, produce biodiesel y abastece a diversas empresas de Morelia para su consumo en vehículos (Moreco 2010).

La planta de producción de biodiesel ubicada en Lázaro Cárdenas, Michoacán, denominada Bioenermex fue inaugurada en 2007. El objetivo inicial fue generar aproximadamente 9,000,000 L de biodiesel al año, además de dar empleo a mil personas de manera inicial. En la actualidad, la planta no se encuentra en operación (Olmedo 2007, Sarmiento 2008, Moreco 2014). El cuadro III resume la información de las plantas instaladas en México.

En el caso de Chiapas, al programa de desarrollo del biodiesel se le denominó Chiapas Bioenergético e incluyó el establecimiento de los cultivos, la extracción del aceite y la construcción y operación de las plantas productoras de biodiesel. Las materias primas seleccionadas para abastecer de aceite a estas plantas fueron Jatropha curcas L., palma africana, higuerilla 


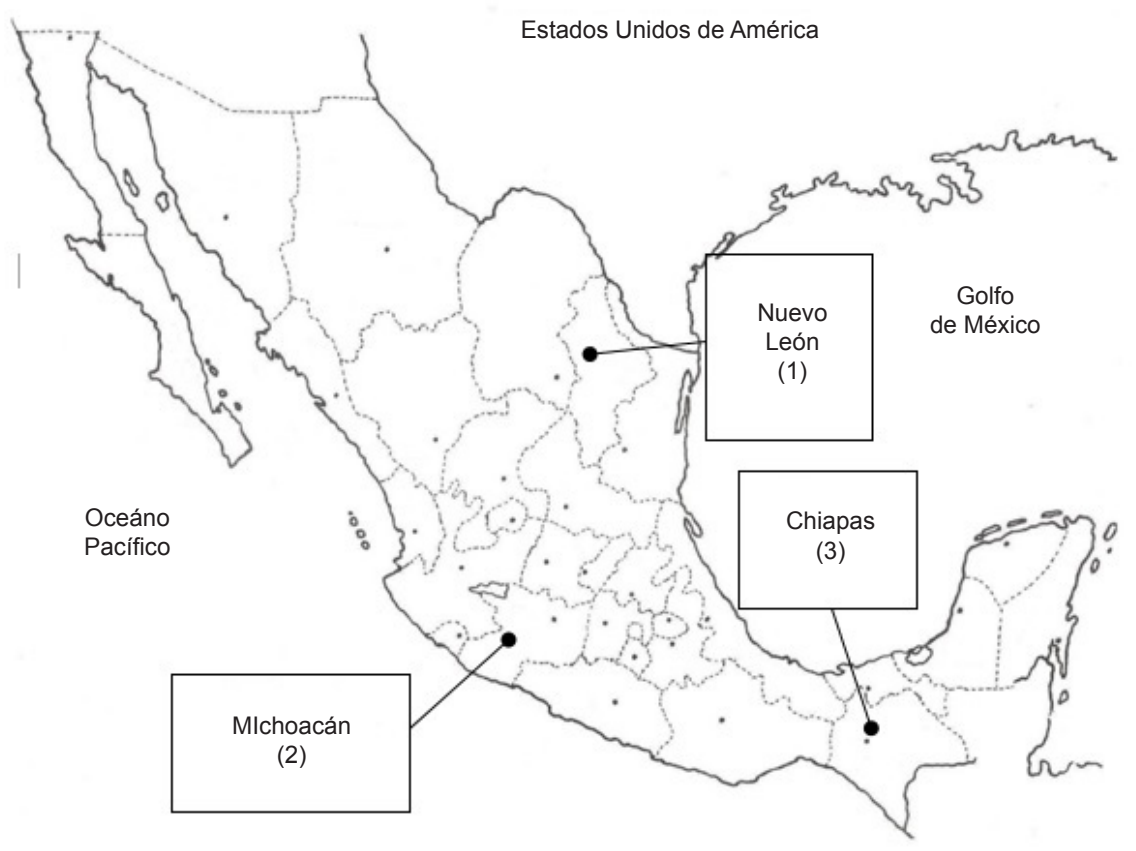

Fig. 6. Ubicación de plantas productoras de biodiesel en México (Elaboración propia)

CUADRO III. INFORMACIÓN DE PLANTAS INSTALADAS EN MÉXICO

\begin{tabular}{|c|c|c|c|}
\hline Ubicación & $\begin{array}{l}\text { Capacidad } \\
(1 / \text { día })\end{array}$ & Materia prima & $\begin{array}{l}\text { Inicio de } \\
\text { operaciones }\end{array}$ \\
\hline Cadereyta, Nuevo León & 50000 & $\begin{array}{l}\text { Sebo de res } \\
\text { AVR }\end{array}$ & 2004 \\
\hline Morelia, Michoacán & No reportada & AVR & 2006 \\
\hline $\begin{array}{l}\text { Lázaro Cárdenas, Michoa- } \\
\text { cán }\end{array}$ & 24600 & Jatropha Curcas & 2007 \\
\hline Tuxtla Gutiérrez, Chiapas & 2000 & $\begin{array}{l}\text { Jatropha Curcas } \\
\text { Palma africana } \\
\text { AVR }\end{array}$ & 2009 \\
\hline Puerto Chiapas & 8000 & $\begin{array}{l}\text { Jatropha Curcas } \\
\text { Palma africana } \\
\text { AVR }\end{array}$ & 2009 \\
\hline Puerto Chiapas & 20000 & $\begin{array}{l}\text { Jatropha Curcas } \\
\text { Palma africana } \\
\text { Higuerilla } \\
\text { AVR }\end{array}$ & 2009 \\
\hline
\end{tabular}

y AVR. La capacidad instalada de producción de biodiesel en Chiapas es de 30,000 1/día, distribuidos en: una planta localizada en Tuxtla Gutiérrez, de tecnología sueca, con una producción de 2000 1/día; otra en Puerto Chiapas constituida por 2 módulos, uno de tecnología colombo-mexicana, con una producción de 8000 1/día y otro módulo de tecnología inglesa, con una producción de 20000 1/día. Las plantas iniciaron operaciones en 2009 y cerraron, por insuficiente suministro de materia prima (Prehn y Cumana 2010).

Cuando las plantas procesan AVR o grasas amarillas para la producción de biodiesel, requieren un pretratamiento que genera una cantidad casi nula de residuos en comparación con la cantidad que se genera cuando procesan semillas oleaginosas. Como ejemplos se presentan las composiciones de 
las semillas de higuerilla y de Jatropha curcas L. La figura 7 (Adaptada de Spangenberg 1935) muestra que el contenido máximo de aceite en la semilla de higuerilla que puede ser aprovechado para la obtención de biodiesel es de $46.19 \%$. El resto se considera residuo de proceso.

En la figura 8 (Adaptada de Uribe 2010, Castro 2009) se detalla la composición de almendra que constituye el $65.60 \%$ de la semilla de Jatropha curcas $L$. y el $34.30 \%$ restante corresponde al pericarpio. El contenido máximo de aceite en la semilla de Jatropha curcas $L$. que puede ser aprovechado para la obtención de biodiesel es de $38.36 \%$. El resto se considera residuo de proceso.

Por citar un caso de los intentos fallidos de producción de biodiesel a nivel industrial en México, Chiapas Bioenergético, evidencia que no es conveniente que la cadena de valor del biodiesel esté a cargo de una sola empresa. Es necesario involucrar a varias empresas responsables en cada etapa, desde la logística y producción de materias primas hasta la comercialización del biocombustible, para que de esta manera, al fallar una de las partes es posible reemplazarla y lograr que la cadena de valor siga funcionando.

Además de los desarrollos a escala industrial, Colombia y México cuentan con proyectos de investigación en marcha para impulsar la producción de biodiesel a partir de AVR y de otros residuales tales como la semilla de café.

\section{OTRAS EXPERIENCIAS EN COLOMBIA}

El Centro Nacional de Investigaciones de Café (CENICAFÉ) de Colombia, se ha dado a la tarea de realizar investigaciones que permitan el aprovechamiento de los residuos del procesamiento del café, para obtener biocombustibles, es decir, biogás, bioetanol y biodiesel. Entre los subproductos aprovechables para la obtención de biodiesel está la borra de café, residuo que se genera en las fábricas de café soluble, el cual corresponde a la fracción insoluble del grano tostado. A partir de este subproducto es posible obtener hasta un $10 \%$ de rendimiento de aceite, el cual es susceptible de ser transformado en biodiesel mediante una reacción de transesterificación (CENICAFÉ 2010).

Entre 2005 y 2011, Colombia utilizó biodiesel de palma en tres pruebas, en tres etapas, que fueron financiadas, por entidades tanto públicas como privadas involucradas en la cadena de biocombustibles. Es así que participaron: el Ministerio de Minas

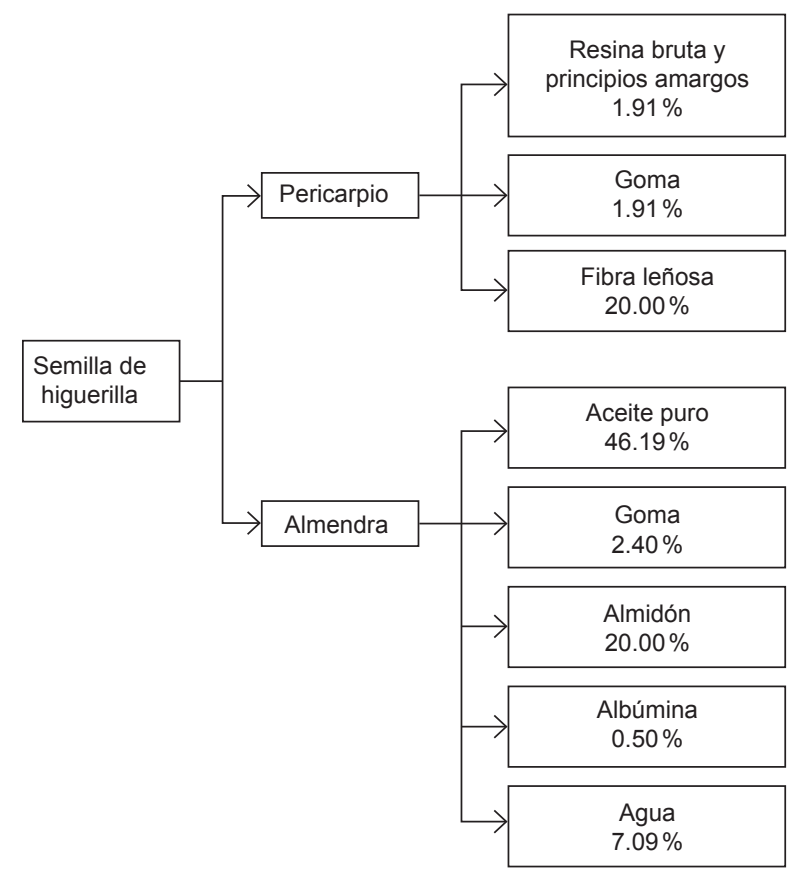

Fig. 7. Composición de la semilla de higuerilla

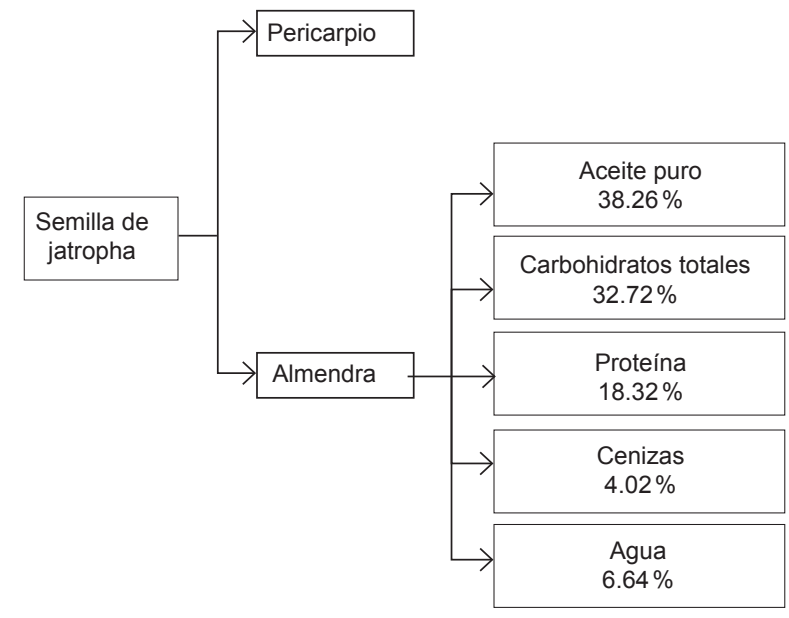

Fig. 8. Composición de la semilla de Jatropha curcas $L$

y Energía, el Instituto Colombiano del Petróleo, FEDEPALMA, CENIPALMA, ECOPETROL, GM Colmotores, Exxonmobil, Shell y la Universidad de Antioquia (FAO 2013).

En 2005, CENIPALMA y el Instituto Colombiano del Petróleo realizaron la caracterización de biodiesel de palma y evaluaron su desempeño en motores diesel a nivel laboratorio.

En 2008, culminaron los recorridos de prueba de un total de $1200000 \mathrm{~km}$, con 12 autobuses que operaron con mezclas diesel-biodiesel al 5\%, 10\%, 
$20 \%, 30 \%$ y $50 \%$. Con base en los resultados, los autobuses utilizan actualmente una mezcla B7.

En 2011, finalizaron los recorridos de prueba de un total de $900000 \mathrm{~km}$ con nueve camiones de carga Chevrolet NKR. Se probaron mezclas hasta del 30\% de biodiesel con un resultado exitoso.

Casi a la par de la promulgación de leyes y mandatos en materia de producción y consumo de biodiesel en Colombia, se inició a nivel de investigación y desarrollo la producción de biodiesel, concluyendo que la materia prima más adecuada era el aceite de palma. Posteriormente, se llevaron a cabo pruebas en motores de combustión interna a nivel laboratorio en centros de investigación con la finalidad de evaluar su desempeño con biodiesel y diversas mezclas dieselbiodiesel. Después se llevaron a cabo estudios de campo para probar el biodiesel de palma y mezclas diesel-biodiesel en autobuses urbanos y de carga en distintas rutas y tiempos prolongados. Estas etapas aseguraron la factibilidad de la utilización del biodiesel en motores diesel. Con base en los resultados obtenidos, escalaron a nivel industrial la producción de biodiesel de palma, la cual continúa de manera exitosa debido a la existencia de un mercado constituido principalmente por el sector transporte.

\section{OTRAS EXPERIENCIAS EN MÉXICO}

En el estado de Oaxaca, México, la Universidad José Vasconcelos cuenta desde 2004 con una planta piloto, para producir biodiesel a partir de AVR, con una capacidad de 45,000 L/año, que se utiliza como B20 en un autobús de la misma universidad (Sarmiento 2008).

En 2005, el Tecnológico de Monterrey construyó una primera planta piloto, con fines de investigación, para producir biodiesel a partir de AVR, con capacidad para producir aproximadamente 240000 L/año (Quintero et al. 2009).

En 2006, la Comisión Estatal de Servicios Públicos de Tijuana (CESPT), Baja California, México, dio inicio al programa: "Atrapa la Grasa", para evitar el envío de grasas y aceites al sistema de alcantarillado municipal. Posteriormente, en 2009, se convirtió en la primera dependencia de Gobierno de este estado, en implementar el consumo de biocombustibles en sus unidades de trabajo con un programa conocido como "Programa de Implementación de Biocombustibles Ecológicos" (CESPT 2007, CESPT 2009a, CESPT 2009b). En 2011, dando continuidad a este proyecto, se firmó un convenio entre la CESPT y la Cámara Nacional de la Industria de Restaurantes y Alimentos
Condimentados, para reforzar el proyecto "Atrapa la Grasa", con la finalidad de reciclar los AVR para la protección del ambiente y su uso como materia prima para la producción de biodiesel (CESPT 2011).

En 2007, en el Instituto de Ingeniería de la Universidad Autónoma de Baja California, se obtuvieron los primeros lotes de biodiesel a partir de AVR. Cuenta con dos reactores para procesar un total de $416 \mathrm{~L}$ de biodiesel por lote. Además de la utilización de AVR, se ha producido biodiesel a partir de aceite de higuerilla, oleaginosa no comestible y propia del Valle de Mexicali. Posteriormente, se han efectuado proyectos de investigación sobre temas relacionados con el inventario de materias primas residuales para producir biodiesel. Para el AVR, se estimó con base en un estudio piloto la generación de 8 millones de litros anuales en el sector restaurantero. En el caso de las grasas amarillas, se efectuaron visitas a las plantas de procesamiento de cárnicos para determinar el porcentaje de grasas por animal procesado. Esta información aunada a la obtenida de las estadísticas oficiales de SAGARPA, permitieron estimar una generación de $1380 \mathrm{t}$ (Toscano et al. 2011, Montero et al. 2011, Vázquez et al. 2011, Montero et al. 2014).

Considerando tales residuos, se realizó un análisis para producir 4.78 millones de litros de biodiesel, energía equivalente a 4.45 millones de litros de diesel. La aplicación del biodiesel se planteó para satisfacer parcialmente la demanda del sector de transporte de carga de Mexicali que en 2010 constó de 11861 unidades con un consumo anual de 169 millones de litros de diesel. Se estimó que el beneficio ambiental debido al reemplazo del diesel con biodiesel, representaría la reducción de aproximadamente de $9700 \mathrm{t}$ de $\mathrm{CO}_{2}, 22 \mathrm{t}$ de $\mathrm{SO}_{2}$ y $11 \mathrm{t}$ de $\mathrm{PM}_{10}$.

Con el biodiesel obtenido a partir de AVR en el laboratorio, se probó el desempeño de un motor KUBOTA D600-B de combustión interna que operó con mezclas diesel-biodiesel (Pérez et al. 2015, Kubota 1995). Asimismo se realizaron estudios sobre el comportamiento de materiales expuestos al diesel, biodiesel y mezclas de ambos (Coronado et al. 2013, Coronado et al. 2014). También se han llevado a cabo investigaciones enfocadas a la producción de biodiesel por vía enzimática (Toscano et al. 2011, Toscano et al. 2012, Toscano et al. 2013, Toscano et al. 2014).

En 2012, la Benemérita Universidad Autónoma de Puebla inició sus primeras pruebas de producción para generar aproximadamente 18000 L/año de biodiesel y actualmente reportan una capacidad de producción de hasta 72000 1/año. La materia prima utilizada fue aceite extraído de semilla de higuerilla. 
La producción estaba destinada al abasto de los camiones del sistema de transporte universitario de la institución. Los fondos fueron otorgados por el Consejo Nacional de Ciencia y Tecnología (CONACYT) y el gobierno estatal (Foro Consultivo Científico y Tecnológico 2014).

En 2012, el Pumabus de Ciudad Universitaria (CU) comenzó a utilizar biodiesel. Esto se dio como resultado de estudios elaborados por estudiantes de la UNAM, quienes determinaron que se generaban más de $10720 \mathrm{~L}$ de AVR anualmente, los cuales eran desechados por los restaurantes de CU (UNAMDGCS 2012).

En 2013, entró en operación una planta de producción de biodiesel en Zacatecas, ubicada en la Universidad Autónoma de Fresnillo, con una inversión de 1.6 millones de pesos. Sin embargo, no se han cumplido las expectativas, pues se esperaba vincular a los sectores productivo y empresarial, pero a la fecha sólo sirve para que los alumnos realicen prácticas. Su meta es llegar a producir $100000 \mathrm{~L}$ de biodiesel semanalmente (Investigación y Desarrollo 2014).

Desde 2014, la empresa Bioregeneradora de Baja California, se dedica a la recolección de aceite vegetal residual del sector restaurantero y a partir de este residuo produce biodiesel para autoconsumo como B100 (Bioregenedora de Baja California 2014).

\section{DISCUSIONES}

El éxito del desarrollo de la industria del biodiesel en Colombia se debe a la integración de los productores de palma quienes proveen las materias primas; los productores de biodiesel; empresarios del sector transporte como consumidores principales; legisladores y gobernantes, responsables de la creación de leyes, reglamentos y mandatos así como de la vigilancia de su cumplimiento; universidades y centros de investigación, dedicados a la investigación y desarrollo tecnológico, pruebas de calidad y desempeño de biodiesel. El trabajo articulado de todos estos actores, provee y asegura la plataforma y condiciones de mercado del biodiesel. El reto para Colombia es continuar con esta dinámica y explorar otras materias primas tales como los AVR y producir biodiesel a partir de éstas.

En México no se ha logrado este nivel de integración de los diversos actores, por lo que el desarrollo de la industria del biodiesel ha quedado rezagado. Los legisladores y gobernantes, representan un eslabón clave, puesto que su trabajo a través del decreto de mandatos que establezcan la obligatoriedad del consumo de biodiesel, aseguraría la demanda y por ende la oferta del biocombustible.

Asimismo, es necesario que se apliquen incentivos para los productores de cultivos bioenergéticos para producir biodiesel en México, debido a que se requieren de 3 o más años para las primeras cosechas. Esto ocasiona que durante los primeros años, los productores no logren obtener ningún ingreso, lo cual desmotiva el sembrado de oleaginosas no comestibles.

El reto para México, es lograr la integración de los actores y establecer mandatos obligatorios para propiciar el mercado del biodiesel.

\section{CONCLUSIONES}

El desarrollo de biodiesel de primera generación en Colombia a escala industrial es una realidad. Prueba de ello, es la existencia de una industria sólida y cadena de valor exitosa, que integra desde los productores de aceite de palma y de biodiesel, hasta su mezcla con diesel y venta en las estaciones de servicio de combustibles para su uso final en vehículos ya sea del sector doméstico o transporte. Esto ha sido posible por las leyes, reglamentos y mandatos vigentes en materia de biocombustibles coordinada con un adecuado manejo de políticas públicas, que fomentan e incentivan la producción y que hacen obligatorio el consumo de biodiesel. En relación con la producción de biodiesel de segunda generación, Colombia muestra sus primeros desarrollos a partir de los residuos del café y otros residuos.

En México, centros de investigación, instituciones de educación superior, inclusive secretarías gubernamentales, cuentan con recursos humanos que poseen las capacidades y aptitudes en lo que concierne a la producción de biodiesel. Sin embargo, ha orientado sus esfuerzos de política energética principalmente hacia la optimización de la industria petrolera y gas natural, y en menor medida a las energías renovables, entre los que se encuentra el biodiesel.

Se han presentado varios casos fallidos de desarrollos industriales de producción de biodiesel de segunda generación en México. Algunos de ellos por la falta del aseguramiento de la disponibilidad de materias primas, otros por la falta de mandatos que establezcan la obligatoriedad del consumo de biodiesel.

México puede aprender de las estrategias y experiencias de Colombia en cuanto al desarrollo de la industria del biodiesel, donde fue necesario decretar la obligatoriedad del consumo de mezclas dieselbiodiesel. Por lo tanto, es necesario que el marco regulatorio de México incluya mandatos obligatorios 
para asegurar el consumo de biodiesel. Asimismo, que establezca metas calendarizadas y porcentajes de biodiesel en estaciones de servicio y se logre la integración de los consumidores potenciales.

Se recomienda que el biodiesel sea de segunda y/o tercera generación con la finalidad de evitar en poner en riesgo la seguridad y soberanía alimentaria del país.

\section{AGRADECIMIENTOS}

Los autores agradecen al Consejo Nacional de Ciencia y Tecnología y al Instituto de Ingeniería de la Universidad Autónoma de Baja California por las facilidades para llevar a cabo el presente proyecto.

\section{REFERENCIAS}

Altin R., Cetinkaya S., Yucesu H.S. (2001). The Potential of Using Vegetable Oil Fuels as Fuel for Diesel Engines. Energy Conversion and Management, 42(5), 529-538.

Bioregenedora de Baja California (2014). Recolección aceite vegetal residual del sector restaurantero. [en línea] http://bioregeneradorabc.com/?page_id=20 06/06/2014.

Boey P., Maniam G. y Hamid S. (2009). Biodiesel from Adsorbed Waste Oil on Spent Bleaching Clay using $\mathrm{CaO}$ as a Heterogeneous Catalyst. European Journal of Scientific Research, 33(2), 347.

Canacki M. (2007). Combustion Characteristics of a Turbocharged DI Compression Ignition Engine Fueled with Petroleum Diesel Fuels and Biodiesel. Bioresource Technology, 98(6), 1167-1 DOI: http://dx.doi. org/10.1016/j.biortech.2006.05.024

Canacki M., Gerpen J.V. (1999). Biodiesel Production Via Acid Catalysis. American Society of Agricultural Engineers, 42(5), 1203-1210. DOI: 10.13031/2013.13285

Castro F. (2009). Diseño de una planta de extracción, semirefinamiento y transesterificación de aceite de jatropha (Jatropha curcas). [en línea] https://bdigital.zamorano.edu/bitstream/11036/1273/1/AGI-2009-T008. pdf 07/04/2015.

CESPT (2007). CESPT reduce derramamiento de grasas al drenaje. Comisión Estatal de Servicios Públicos de Tijuana. [en línea] http://www.cespt.gob.mx/ServNoticias/VerNoticia.aspx?id=50 12/03/2014.

CESPT (2009a). CESPT pionero en la utilización de biocombustibles ecológicos. Comisión Estatal de Servicios Públicos de Tijuana. [en línea] http://www. cespt.gob.mx/ServNoticias/VerNoticia.aspx?id=130 $12 / 03 / 2014$.

CESPT (2009b). CESPT utilizará biocombustible en sus unidades. Comisión Estatal de Servicios Públicos de Tijuana. [en línea] http://www.cespt.gob.mx/ServNoticias/VerNoticia.aspx?id=128 12/03/2014.

CESPT (2011). Continua CESPT promoviendo programa "Atrapa la Grasa" en CANIRAC. Comisión Estatal de Servicios Públicos de Tijuana. [en línea] http://www. cespt.gob.mx/ServNoticias/VerNoticia.aspx?id=371 $12 / 03 / 2014$.

CENICAFÉ (2010). Avances técnicos 393, Marzo. Centro Nacional de Investigaciones de Café. [en línea] http://www.cenicafe.org/es/publications/avt0393.pdf 13/03/2014.

Coronado M., Montero G., Eliezer A., García C., Cerezo J., Pérez L. y Ayala J. (2013). Materials technological challenges for the biodiesel industry development in Mexico. En: Materials and processes for energy: communicating current research and technological developments, Editorial Formatex Research Center, pp. 279-288. [en línea] http://www.formatex.info/ energymaterialsbook/book/279-288.pdf 01/12/2014.

Coronado M., Montero G., Valdez B., Stoytcheva M., Eliezer A., García C., Campbell H. y Pérez A. (2014). Degradation of nitrile rubber fuel hose by biodiesel use, Energy, 68, 364- 369.

DOI: $10.1016 /$ j.energy.2014.02.087.

DOF (2008). Ley de Promoción y desarrollo de los bioenergéticos. Diario Oficial de la Federación. [en línea] www.diputados.gob.mx/LeyesBiblio/pdf/LPDB.pdf 22/06/2014.

DOF (2009). Reglamento de la Ley de Promoción y Desarrollo de los Bioenergéticos. Diario Oficial de la Federación. [en línea] www.diputados.gob.mx/LeyesBiblio/regley/Reg_LPDB.doc 17/06/2014.

Duffey A. (2011). Estudio regional sobre economía de los biocombustibles 2010: temas clave para los países de América Latina y el Caribe. [en línea] http://www. cepal.org/ddpe/agenda/2/42932/esteconomiabiocombustiblesdialpol.pdf 23/06/2014.

EL ECONOMISTA (2011). Fracasa proyecto de biodiesel. [en línea] http://eleconomista.com.mx/estados/2011/07/18/fracasa-proyecto-biodiesel. 23/03/2014.

FAO (2013). La bioenergía en América Latina y El Caribe, El estado de arte en países seleccionados. Food and Agriculture Organization. [en línea] http://www.fao. org/docrep/019/as112s/as112s.pdf 23/03/2015.

FEDEPALMA (2002). Guía ambiental para el subsector de la palma de aceite. Federación Nacional de Cultivadores de Palma de Aceite. [en línea] http://www. siame.gov.co/siame/documentos/Guias_Ambientales/ Gu\%C3\%ADas\%20Resoluci\%C3\%B3n\%201023\%20 del $\% 2028 \% 20$ de $\% 20$ julio $\% 20$ de $\% 202005 /$ AGRICOLA $\% 20$ Y\%20PECUARIO/Gu\%C3\%ADa\%20 ambiental\%20para $\% 20$ el $\% 20$ subsector $\% 20$ Palma $\% 20$ 
de $\% 20$ Aceite.pdf 26/03/2015.

FEDEBIOCOMBUSTIBLES (2014). Información Estadística Sector Biocombustibles. Federación Nacional de Combustibles de Colombia. [en línea] http://www. fedebiocombustibles.com/v3/estadistica-mostrar_infotitulo-Biodiesel.htm 05/06/2014.

FORO CONSULTIVO CIENTÍFICO Y TECNOLÓGICO (2014). Contará la BUAP con planta de producción de biodiesel. [en línea] http://www.foroconsultivo.org. $\mathrm{mx} /$ innovacion.gaceta/component/content/article/224-innovadores-/303-contara-la-buap-con-plantade-produccion-de-biodisel 01/08/2014.

Gaceta Parlamentaria (2014). Cámara de Diputados, México. Iniciativa que reforma y adiciona diversas disposiciones de la ley de promoción y desarrollo de los bioenergéticos suscrita por el diputado Javier Orihuela García, del PRD, y por integrantes de diversos grupos parlamentarios. Año XVIII, núm. 4176-X, Lunes 15 de diciembre de 2014. [en línea] www.gaceta.diputados. gob.mx 07/01/2015.

Gerpen J.V., Pruszko R., Clements D., Shanks B., Knothe G. (2006). Building a successful biodiesel business. Biodiesel basics. Second edition. Idaho, EUA, 278 pp.

Graboski M.S, McCormick R.L. (1998). Combustion of Fat and Vegetable Oil Derived Fuels in Diesel Engines. Progress in Energy and Combustion Science, 24(2), 125-164. DOI:10.1016/S0360-1285(97)00034-8

IEA (2014). Biofuels Outlook market developments and policy challenges. International Energy Agency. [en línea] http://www.ieabioenergy.com/wp-content/ uploads/2014/05/P01-Biofuels-Outlook-marketdevelopments-and-policy-challenges-Eisentraut.pdf 01/04/2015.

INVESTIGACIÓN Y DESARROLLO (2014). Sin "clientes" laboratorio de biodiesel en zacatecas. [en línea] http://www.invdes.com.mx/ciencia-mobil/5074sin-clientes-laboratorio-de-biodiesel-en-zacatecas $15 / 08 / 2014$.

IRENA (2013). Road transport: the cost of renewable solutions. International Renewable Energy Agency. [en línea] www.irena.org/.../Publications/Road_Transport. pdf 29/03/2015.

Iscan B. y Aydin H. (2012). Improving the Usability of Vegetable Oil as Fuel in a Low Heat Rejection Diesel Engine. Fuel Process Technol. 98, 59-64. http://dx.doi. org/10.1016/j.fuproc.2012.02.001

Kubota (1995). Workshop Manual Diesel Engine Kubota for Engine Models: Z400-B. Z430-B. D600-B. D640-B V800-B, Kubota. Osaka. Japan. Editor: Kubota Farm \& Industrial Machinery Service. Printed in USA.

Lin Y.C, Lee W.J, Hou H.C. (2006). PAH Emissions and Energy Efficiency of Palm Biodiesel Blends Fueled on Diesel Generator Atmos. Environ. 40(21), 3930-3940. http://dx.doi.org/10.1016/j.atmosenv.2006.02.026

Ministerio de Agricultura y Desarrollo Rural, República de Colombia (2012). Política nacional de biocombustibles en Colombia. [en línea] http://www.olade.org/ sites/default/files/seminarios/BIO2012/PONENCIAS/ Sesion\%201_J\%20Mejia_Colombia.pdf 23/06/2014.

Montero G., Stoytcheva M., García C., Coronado M., Toscano L., Campbell H., Pérez A. y Vázquez A. (2011). Current status of biodiesel production in Baja California, Mexico. (G. Montero, Ed.). En: Biodiesel quality, emissions and by- products. Editorial InTech. pp. 137-152. [en línea]. DOI: 10.5772/27159

Montero G., Coronado M., Campbell H. Cerezo, J., Lambert A. y Valenzuela E. (2014). Biomass Wastes: An Energy Option for Baja California, México. J. of Power and Energ. Eng. 2, 146-150.

DOI: $10.4236 /$ jpee.2014.24021.

MORECO (2010). Clientes Moreco. [en línea] http://www. moreco.com.mx/clientes/ 03/04/2014.

MORECO (2014). Visita a planta de biodiesel en L.C. Michoacán. [en línea] http://www.moreco.com.mx/ nosotros/ 30/05/2014.

Nielsen P., Brask J. y Fjerbaek L. (2008). Enzymatic Biodiesel Production: Technical and Economical consideration. Eur. J. Lipid. Sci. Tech. 110(8), 692-700. DOI: 10.1002 /ejlt.200800064

Olmedo F. (2007). Inauguran primera planta productora de biodiesel en Michoacán. [en línea]. http://www.biodisol.com/biocombustibles/inauguran-primera-plantaproductora-de-biodiesel-en-michoacan 28/05/2014.

Pérez-Sánchez A., Montero-Alpírez G., Ayala-Bautista J.R., Coronado-Ortega M.A., García-González C. y Campbell-Ramírez H.E. (2015). Simulación en Aspen de la combustión de mezclas diesel-biodiesel. Ingeniería Investigación y Tecnología. XVI(1), 83-92. http:// dx.doi.org/10.1016/S1405-7743(15)72109-8

Prehn M. y Cumana I. (2010). La Bioenergía en México: estudios de caso $\mathrm{n}^{\circ} 1$. [en línea] http://www.rembio.org.mx/2011/Documentos/Cuadernos/CT1.pdf $19 / 05 / 2014$.

Quintero L., Guerra R., Llamas A. (2009). Producción de biodiesel en el Centro de Estudios de Energía del ITESM Campus Monterrey. Ide@s CONCYTEG. [en línea] http://www.concyteg.gob.mx/ideasConcyteg/ Archivos/43032008_PROD_BIODIESEL_CENTRO_ ESTUDIOS_ENERGIA_ITESM.pdf 21/12/2016.

Rakopoulos C.D., Antonopoulos K.A., Rakopoulos D.C., Hountalas D.T. y Giakoumis E.G. (2006). Comparative Performance and Emissions Study of a Direct Injection Diesel Engine Using Blends of Diesel Fuel with Vegetable Oils or Biodiesels of Various Origins. Energ. Convers. Manage. 47 (18-19), 3272-3287. http:// dx.doi.org/10.1016/j.enconman.2006.01.006 
Refaat A. (2011). Biodiesel Production Using Solid Metal Oxide Catalysts. Int. J. Environ. Sci. Technol. 8 (3), 621-630. DOI:10.1007/BF03326210

Reinosa D. (2009). Costos ambientales en el proceso de extracción del aceite de palma. Estudio de un caso. Revista Venezolana de Gerencia, 14(46), 228-247. Universidad del Zulia Venezuela.

Sarmiento R. (2008). Primeros desarrollos de producción de biodiesel en México. [en línea]. http://energiaadebate.com/Articulos/Julio2008/RocioSarmientoJulio2008.htm 06/05/2014.

Spangenberg G. (1935). El cultivo del ricino. Revista Chilena de Historia Natural. [en línea] http://rchn. biologiachile.cl/pdfs/1935/1/Spangenberg_1935.pdf 03/04/2015.

Toscano L., Gochev V., Montero G. y Stoytcheva M. (2011). Enhanced production of extracellular lipase by novel mutant strain of aspergillus niger. Biotechnol. Biotec. Eq. 25 (1), 2243-2247, DOI: $10.5504 /$ bbeq. 2011.0019

Toscano L., Montero G., Stoytcheva M., Gochev V., Cervantes L., Campbell H., Zlatev R., Valdez B., Pérez C. y Samaniego M. (2012). Lipase production through solid-state fermentation using agro-industrial residues as substrates and newly isolated fungal strains. Biotechnol. Biotec. Eq. http://dx.doi.org/10.5504/ BBEQ.2012.0145

Toscano L., Montero G., Cervantes L., Stoytcheva M., Gochev V. y Beltrán M. (2013). Production and partial characterization of extracellular lipase from trichoderma harzianum by solid-state fermentation. Biotechnol. Biotec. Eq. 27(2), 3776-3781. http:// dx.doi.org/10.5504/BBeQ.2012.0140
Toscano L., Montero G., Stoytcheva M., Cervantes L. y Gochev V. (2014). Comparison of the performances of four hydrophilic polymers as supports for lipase immobilisation. Biotechnol. Biotec. Eq. 28 (1), 5260. http://dx.doi.org/10.1080/13102818.2014.901684

Toscano L., Montero G., Stoytcheva M. y Campbell H. (2011). Preliminary assessment of biodiesel generation from meat industry residues in Baja California, Mexico. Biomass Bioenerg. 35 (1), 26-31.

DOI: $10.1016 /$ j.biombioe.2010.10.031

Trejo E. (2007). Estudio de derecho comparado y marco jurídico internacional sobre biocombustibles/bioenergéticos. [en línea] www.diputados.gob.mx/sedia/sia/ spe/SPE-ISS-08-07.pdf 23/05/2014.

UNAM-DGCS (2012). Donan planta de biodiesel a la Facultad de Ingeniería de la UNAM. [en línea]. http:// www.dgcs.unam.mx/boletin/bdboletin/2012_670.html 20/05/2014.

Uribe (2010). Simulación de una planta piloto para la producción de biodiesel en el laboratorio de operaciones unitarias de la ESIQIE. [en línea]. www.riieeme.mx/ docs/tesismoraymabiodiesel2.pdf 01/04/2015

Vázquez A., Montero G., Sosa J., García C. y Coronado M. (2011). Economic Analysis of Biodiesel Production from Waste Vegetable Oil in Mexicali, Baja California. Energ. Sci. Technol. 1(1), 87-93. http://dx.doi. org $/ 10.3968 \% 2 \mathrm{Fi} 93$

Vyas A., Berma J. y Subrahmanyam N. (2010) A Review on FAME Prod. Processes. Fuel. 89 (1), 1-9. http:// dx.doi.org/10.1016/j.fuel.2009.08.014 